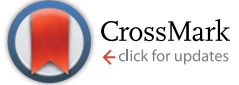

Cite this: RSC Adv., 2017, 7, 5966

Received 15th November 2016 Accepted 7th January 2017

DOI: 10.1039/c6ra26817j

www.rsc.org/advances

\section{Stable ambipolar organic-inorganic heterojunction field-effect transistors and inverters with Cytop interlayer $\dagger$}

\author{
Sheng Sun, Linfeng Lan, * Yuzhi Li, Honglong Ning, Rihui Yao, Lei Wang \\ and Junbiao Peng
}

In this study, ambipolar field-effect transistors (FETs) based on organic-inorganic bilayer structures were investigated. $\mathrm{InO}_{x}$ and pentacene were selected as $\mathrm{n}$-type and $\mathrm{p}$-type semiconductors, respectively. To improve the hole mobility, poly(perfluorobutenylvinylether) (Cytop) layer was introduced to modify the surface of $\operatorname{lnO}_{x}$. The ambipolar FETs showed hole and electron mobilities of 1.1 and $0.1 \mathrm{~cm}^{2} \mathrm{~V}^{-1} \mathrm{~s}^{-1}$, respectively and excellent electrical stability under gate bias stress. Furthermore we found that ambipolar FETs could be integrated into functional complementary metal oxide semiconductor (CMOS)-like inverters and exhibited high peak gain $(>50)$. This work provides a general method for realizing ambipolar FETs based on organic-inorganic hybrid structure.

\section{Introduction}

In recent years, ambipolar field-effect transistors (FETs) have been extensively studied for technological applications in complementary logic circuits due to their unique operation, which allows both holes and electrons to be injected and transported depending on the gate bias in a single device..$^{1-5}$ Organic semiconductors and metal-oxide semiconductors are two families of active materials that are currently drawing much attention in FETs. ${ }^{6-8}$ However, they usually exhibit unipolar charge transport, which limits their applications in ambipolar FETs. Although organic semiconductors can transport both holes and electrons at the same time, the electron transport for most of the organic semiconductor is poor compared to the hole transport. ${ }^{9}$ In contrary to the organic semiconductors, most metal-oxide semiconductors have only n-type characteristics. ${ }^{10,11}$ Therefore, it is possible to achieve ambipolar transport by using organic semiconductor/oxide semiconductor bilayer structure. Nakanotani et al. developed an ambipolar FET based on an organic-inorganic hybrid structure containing indium zinc oxide and pentacene bilayer with hole and electron mobilities of 0.14 and $13.8 \mathrm{~cm}^{2} \mathrm{~V}^{-1} \mathrm{~s}^{-1}$, respectively. ${ }^{12}$ This work opened up a new field in ambipolar FETs fabrication. Smith et al. reported an air-stable solution-processed hybrid transistors with hole and electron mobilities exceeding $2 \mathrm{~cm}^{2} \mathrm{~V}^{-1} \mathrm{~s}^{-1}$, but the device showed ambipolar performance only in the dual-

State Key Laboratory of Luminescent Materials and Devices (South China University of Technology), Wushan Road 381\#, Tianhe District, Guangzhou, China. E-mail: lanlinfeng@scut.edu.cn

$\dagger$ Electronic supplementary information (ESI) available. See DOI: $10.1039 / \mathrm{c} 6 \mathrm{ra} 26817 \mathrm{j}$ gated model, which is complicated and costly. ${ }^{13}$ Yang et al. reported ambipolar FETs based on pentacene/ZnO hybrid structures and got balance hole and electron mobilities of 0.34 and $0.38 \mathrm{~cm}^{2} \mathrm{~V}^{-1} \mathrm{~s}^{-1}$ respectively. ${ }^{14}$ Kim et al. reported ambipolar FETs based on carbon nanotube/zinc tin oxide heterostructures by inkjet printing with hole and electron mobilities exceeding 2 $\mathrm{cm}^{2} \mathrm{~V}^{-1} \mathrm{~s}^{-1}$, but the processing temperature is as high as $500{ }^{\circ} \mathrm{C}$, which was too high to fabricate on most of the flexible substrates..$^{15}$ Ambipolar FETs have also been realized by several approaches, such as bilayer and blend. But most work can't exhibit good stability. ${ }^{16-20}$

Herein, we report on a high-stable ambipolar FET based on an organic-inorganic bilayer structure channel. For the p-type organic semiconductor, we chose pentacene which was well studied and exhibit stable performance. $\mathrm{InO}_{x}$ was selected as the n-type metal oxide semiconductor. In our previous work, directly patternable solution-processed $\mathrm{InO}_{x}$ films could be fabricated with an economic and environmentally friendly approach at low temperature. ${ }^{21}$ To improve the p-type performance, the surface of the $\mathrm{InO}_{x}$ layer was modified by Cytop. ${ }^{22}$

\section{Experimental section}

Fig. 1 shows the schematic structure of the ambipolar FET. Firstly, a $300 \mathrm{~nm}$ thick Al-Nd (3 wt\%) film was deposited with DC sputtering and patterned with conventional photolithography method. Afterwards, Al:Nd film was anodized for $1.5 \mathrm{~h}$ to produce a layer of $200 \mathrm{~nm}$ thick $\mathrm{AlO}_{x}: \mathrm{Nd}\left(41 \mathrm{nF} \mathrm{cm}^{-2}\right)$ on the surface of the Al:Nd film as the gate insulator. After $5 \mathrm{~min}$ oxygen plasma treatment, the $\mathrm{InO}_{x}$ film was spin-coated and patterned in a facile method, following which the $\mathrm{InO}_{x}$ film underwent an annealing process at $220^{\circ} \mathrm{C}$ for $2 \mathrm{~h}$ in air. After 


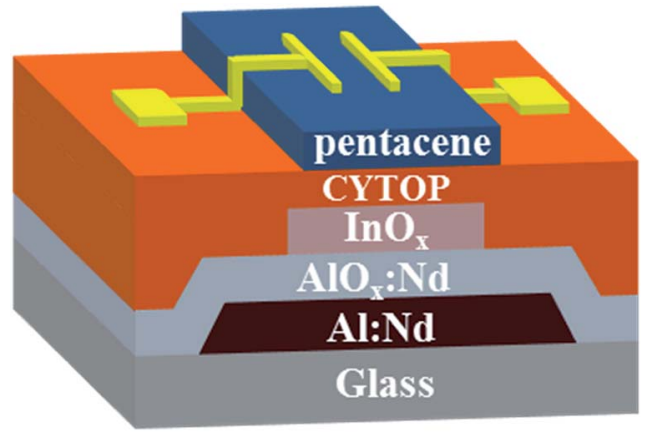

Fig. 1 Schematic diagram of ambipolar FETs based on $\ln O_{x} / C y t o p /$ pentacene hybrid structure.

that, the $45 \mathrm{~nm}$ thick Cytop film was spin-coated as an interlayer to modify the growth of pentacene. After that, a layer of $30 \mathrm{~nm}$ thick pentacene film was thermally evaporated onto the insulator at room temperature under a vacuum pressure of $\sim 3 \times$ $10^{-4} \mathrm{~Pa}$ with a deposition rate of $0.1 \AA^{-1}$. Then a $45 \mathrm{~nm}$ thick $\mathrm{Au}$ film was thermally evaporated onto the pentacene film through a shadow mask, defining a channel width/length $(\mathrm{W} / \mathrm{L})$ of 500/70 $\mu \mathrm{m}$. The TFT characterizations were measured with a semiconductor parameter analyzer (Agilent 4155C) and a probe station at room temperature (RT) in vacuum environment.

\section{Results and discussion}

Fig. 2 shows the output and transfer characteristics of the ambipolar FETs based on the organic-inorganic heterostructures with the Cytop layer. The FETs exhibited ambipolar performance with hole and electron mobilities of 1.1 and 0.1 $\mathrm{cm}^{2} \mathrm{~V}^{-1} \mathrm{~s}^{-1}$, respectively, which were extracted from the following equation,
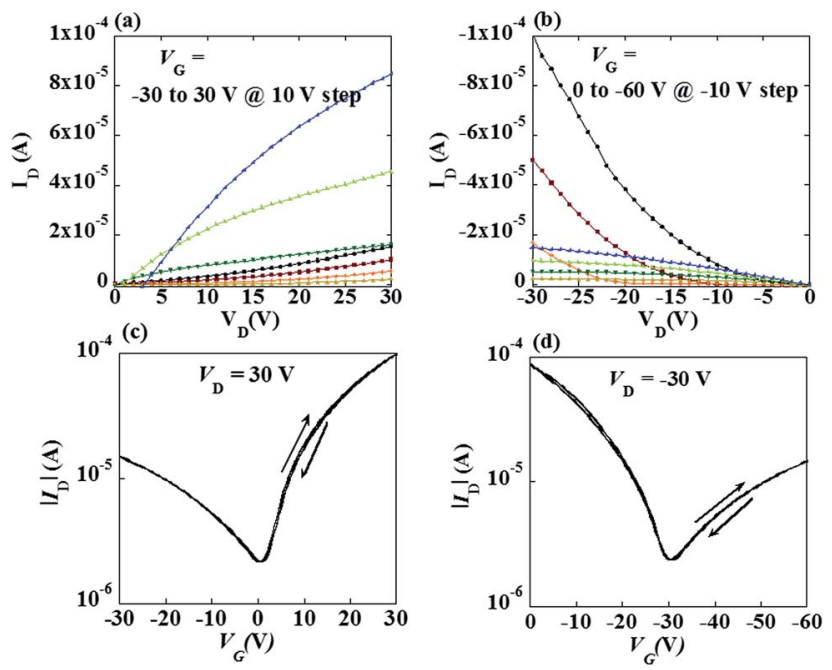

Fig. 2 Output characteristics of ambipolar FETs based on $\ln \mathrm{O}_{x} /$ Cytop/ pentacene hybrid structure: (a) in electron enhancement mode and (b) in electron enhancement mode. Transfer characteristics of ambipolar

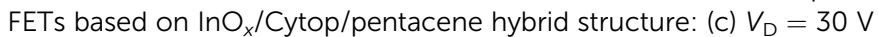
and (d) $V_{D}=-30 \mathrm{~V}$. Every curve includes forward and reverse sweeps.

$$
I_{\mathrm{DS}}=\mu \frac{W}{2 L} C_{\mathrm{i}}\left(V_{\mathrm{G}}-V_{\mathrm{T}}\right)^{2},
$$

where $I_{\mathrm{DS}}$ is drain-source current, $V_{\mathrm{G}}$ is gate voltage, $V_{\mathrm{T}}$ is the threshold voltage, $C_{\mathrm{i}}$ is capacitance density. Specifically, $C_{\mathrm{i}}(22$ $\left.\mathrm{nF} \mathrm{cm}{ }^{-2}\right)$ for hole mobility and $C_{\mathrm{i}}\left(41 \mathrm{nF} \mathrm{cm}{ }^{-2}\right)$ for electron mobility were different, because the $20 \mathrm{~nm}$ thick $\mathrm{InO}_{x}$ and $45 \mathrm{~nm}$ thick Cytop layer were acted as the dielectric layers when the FET was operated in the p-channel mode, as shown in Fig. 3. $C_{\mathrm{i}}$ for hole mobility was constant as gate voltage varies from $0 \mathrm{~V}$ to $-30 \mathrm{~V}$, as shown in Fig. S3. $\dagger$

It could be also seen from Fig. 2 the hysteresis between the forward and reverse sweeps in the transfer curve is too little to be detected. It implies that the amount of fast traps in the bulk or at the interface is small. It is worth noting that the ambipolar FETs without Cytop or with $20 \mathrm{~nm}$ Cytop exhibited large hysteresis, as shown in Fig. S1, $\dagger$ which was attributed to the large amount of hole traps on the surface of $\mathrm{InO}_{x}$ (and $20 \mathrm{~nm}$ Cytop was not thick enough to block the holes from trapping into $\mathrm{InO}_{x}$ ). Besides Cytop, polymethyl methacrylate (PMMA) and polystyrene (PS) were also employed as the modification layer. It can be seen from Fig. S2 $\uparrow$ that the PMMA and PS layer can also improve FET performance, but not as good as Cytop. ${ }^{23,24}$ Compared to PMMA and PS, Cytop film is smoother and more hydrophobic. Besides, the density of states (DOS) of Cytop film is low due to the low dielectric constant, resulting in higher mobility and better stability.

Fig. 4(a) and (b) show the atomic force microscopy (AFM) images of pentacene films on $\mathrm{InO}_{x}$ and Cytop/InO$x$, respectively. The pentacene grain size on $\mathrm{InO}_{x}$ was much larger than that on Cytop/InO ${ }_{x}$. The grain size was based on surface roughness and surface energy. It is known that the pentacene film tends to grow into larger size on the surface of low roughness and high surface energy. ${ }^{25}$ The roughness of $\mathrm{InO}_{x}(1.2 \mathrm{~nm})$ is similar as that of Cytop (1.4 nm), tested by AFM. The surface energy of $\operatorname{InO}_{x}\left(45.6 \mathrm{mN} \mathrm{m}^{-1}\right)$ is larger than that of Cytop $\left(13.5 \mathrm{mN} \mathrm{m}^{-1}\right)$, which was calculated by OWRK (Owens-Wendt-Rabel-Kaelbe) equation using contact angles of water, ethylene glycol and diiodomethane on the surface of $\mathrm{InO}_{x}$ and Cytop. ${ }^{26,27}$ Although the pentacene grain size on $\mathrm{InO}_{x}$ is larger than that on Cytop/ $\mathrm{InO}_{x}$, the hole transport of the FETs without Cytop can hardly be

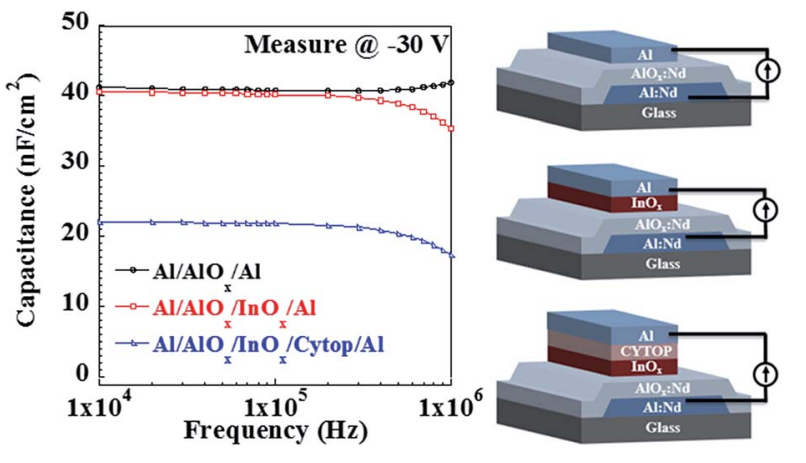

Fig. 3 Capacitance $v s$. frequency plots measured at $-30 \vee$ for $\mathrm{AlO}_{x}: \mathrm{Nd}, \mathrm{AlO}_{x}: \mathrm{Nd} / \mathrm{InO}_{x}$ and $\mathrm{AlO}_{x}: \mathrm{Nd} / \mathrm{InO}_{x} / \mathrm{Cytop}$; inset: the structures of these three capacitances. 

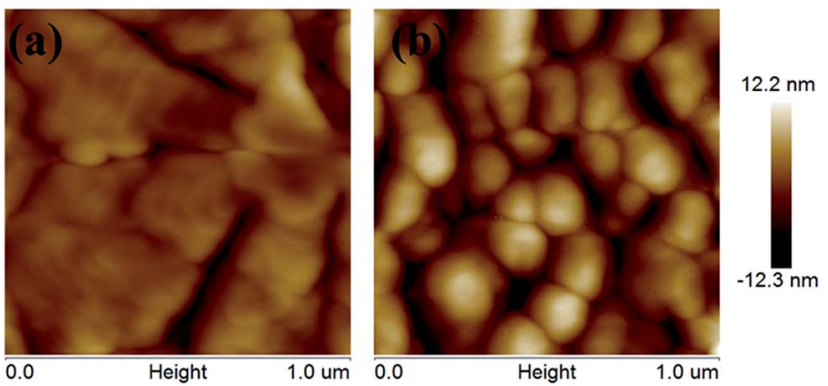

Fig. 4 AFM images of pentacene films on (a) $\ln O_{x}$, and (b) Cytop/lnO

detected. This phenomenon was ascribed to the high electron density of InO $_{x}$. In FETs without Cytop, the large amount of the undepleted electrons can act as the hole traps, so the holes in the organic semiconductor are trapped. After inserted with a Cytop interlayer, the undepleted electrons from $\operatorname{InO}_{x}$ are blocked by Cytop, so the holes in pentacene cannot be trapped by the electrons from $\mathrm{InO}_{x}$. As a result, the hole transport of the FETs with Cytop layer improves.

The electrical stability under gate bias stress is an important parameter in FETs. Fig. 5 and 6 show negative bias stress (NBS) and positive bias stress (PBS) stability of the ambipolar FETs, respectively. Almost no threshold voltage shift was found during the PBS test, while a little positive shift was found during the NBS test. The high bias stability was ascribed to the Cytop modification layer. For electron transport, the Cytop acted as the passivation layers to prevent the back channel of $\mathrm{InO}_{x}$ from interacting with oxygen and moisture from the air. For hole transport, the Cytop layer can passivated the $\mathrm{OH}$ groups on the $\mathrm{InO}_{x}$ surface. It is known that the $\mathrm{OH}$ groups at the insulator/ semiconductor interface would cause device instability. There are no $\mathrm{OH}$ groups in the Cytop molecule, and the Cytop films are highly hydrophobic with a water contact angle of $106^{\circ}$ which is better than those of many other polymers. Thus, the Cytop modification will improve the electrical stability for both electron and hole transport. ${ }^{22}$

Finally, a complementary-like inverter was fabricated with the integration of two identical pentacene/Cytop/ $\mathrm{InO}_{x}$ ambipolar FETs. Fig. 7(a) shows the voltage transfer characteristics. The input voltage was applied through the Al:Nd bottom gate
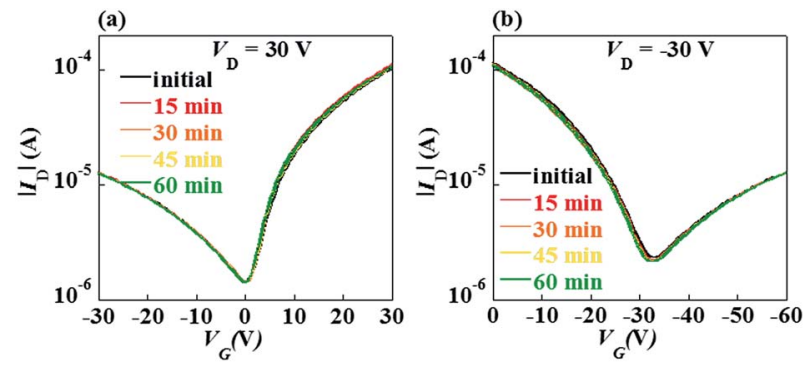

Fig. 5 The time-dependent transfer property ((a) $V_{D}=30 \mathrm{~V}$ and (b) $V_{D}$ $=-30 \mathrm{~V})$ under negative gate-bias-stress for ambipolar FETs stress condition: $V_{G}=30 \mathrm{~V}, V_{D}=30 \mathrm{~V}$. Every curve includes forward and reverse sweeps.
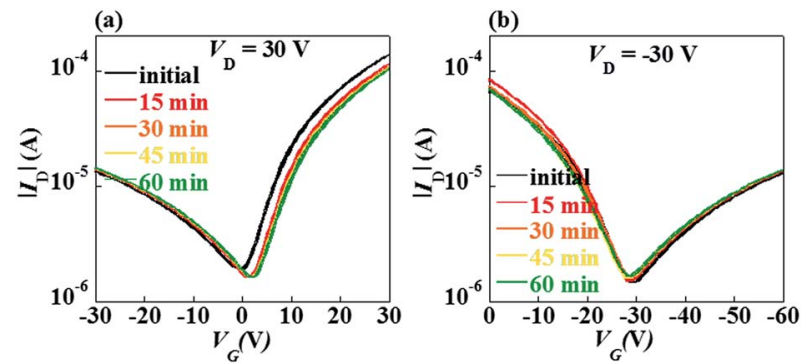

Fig. 6 The time-dependent transfer property ((a) $V_{D}=30 \mathrm{~V}$ and (b) $V_{D}$ $=-30 \mathrm{~V})$ under negative gate-bias-stress for ambipolar FETs stress condition: $V_{G}=-30 \mathrm{~V}, V_{D}=-30 \mathrm{~V}$. Every curve includes forward and reverse sweeps.
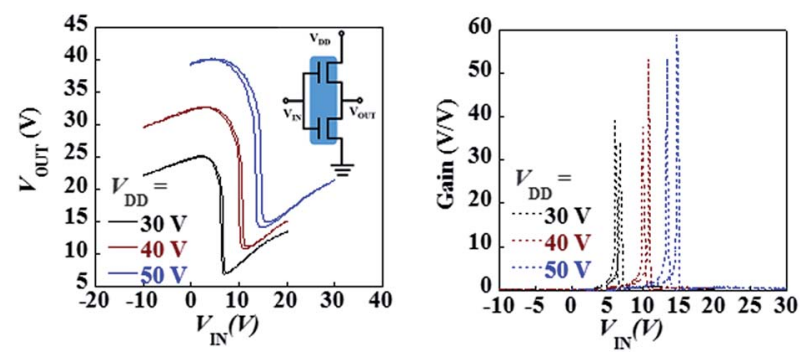

Fig. 7 (a) The voltage transfer characteristics of the CMOS-like inverter at different supply voltages of $30 \mathrm{~V}, 40 \mathrm{~V}, 50 \mathrm{~V}$. Inset: the circuit diagram of the inverter. (b) The corresponding voltage gains of the inverter.

electron. Three supply voltages $\left(V_{\mathrm{DD}}\right)$ of $30 \mathrm{~V}, 40 \mathrm{~V}$ and $50 \mathrm{~V}$ were biased to the inverter respectively. The output voltage $\left(V_{\text {OUT }}\right)$ varied from high to low in response to the swing of the $V_{\mathrm{IN}}$ from low to high, and sharp inversions were found to be near $5 \mathrm{~V}$, $10 \mathrm{~V}$ and $15 \mathrm{~V}$, respectively. The voltage grain, defined as $\mid \mathrm{d} V_{\text {OuT }} /$ $\mathrm{d} V_{\mathrm{IN}} \mid$ was calculated from the voltage transfer curves, as shown in Fig. 7(b). The voltage gains reached maximum value of 40, 54 and 58. The $V_{\text {OUT }}$ was not constant at both high and low values of $V_{\mathrm{IN}}$, where $V_{\mathrm{OUT}}$ increased slightly with $V_{\mathrm{IN}}$. This common feature of ambipolar inverters arose because one of the ambipolar FETs is not completely turned off while the second FET was turned on. ${ }^{15}$ Overall, the inverter exhibited better electrical stability and voltage gain compared to most of other inverters based on unipolar p-type and n-type FETs and ambipolar FETs. ${ }^{28-30}$

\section{Conclusions}

In conclusion, the stable ambipolar FETs and CMOS-like inverters based on organic-inorganic heterostructures were fabricated. Pentacene and $\mathrm{InO}_{x}$ as the semiconducting p-type and n-type layers composed the hybrid structure. Cytop was inserted to modify the organic-inorganic interface by reducing the surface energy to improve the hole mobility and the stability. As a result the ambipolar FETs showed hole and electron mobilities of 1.1 and $0.1 \mathrm{~cm}^{2} \mathrm{~V}^{-1} \mathrm{~s}^{-1}$, respectively and excellent electrical stability under gate bias stress. COMS-like 
inverters showed clear signal inverting operation with a high gain of 58. These results suggest that FETs based on organicinorganic heterostructures are promising candidates for realizing high stable ambipolar logic devices.

\section{Acknowledgements}

This work was supported by the National Key Research and Development Program of China (Grant no. 2016YFB0401105), the National "973" Project of China (Grant no. 2015CB655000), the National Natural Science Foundation of China (Grant no. 51673068, 61204087, and 51173049), the Pearl River S\&T Nova Program of Guangzhou (Grant no. 2014J2200053), the Guangdong Province Science and Technology Plan (Grant no. 2013B010403004, 2014B010105008, 2014B090916002, and 2015B090914003).

\section{Notes and references}

1 A. Dodabalapur, H. E. Katz, L. Torsi and R. C. Haddon, Science, 1995, 269, 1560.

2 J. Zaumseil and H. Sirringhaus, Appl. Phys. Lett., 2007, 107, 1296.

3 B. B. Y. Hsu, E. B. Namdas, J. D. Yuen, S. Cho, I. D. W. Samuel and A. J. Heeger, Adv. Mater., 2010, 24, 4649.

4 Y. Zhao, Y. Guo and Y. Liu, Adv. Mater., 2013, 25, 5372.

5 S. Watanabe, T. Fujita, J. Ribierre, K. Takaishi, T. Muto, C. Adachi, M. Uchiyama, T. Aoyama and M. Matsumoto, ACS Appl. Mater. Interfaces, 2016, 8, 17574.

6 H. Sirringhaus, Adv. Mater., 2014, 26, 1319.

7 B. Kumar, B. K. Kaushik and Y. S. Negi, Polym. Rev., 2014, 54, 3.

8 E. Fortunato, P. Barquinha and R. Martins, Adv. Mater., 2012, 24, 2945.

9 Y. Zhao, Y. Guo and Y. Liu, Adv. Mater., 2013, 25, 5372.

10 K. Nomura, T. Kamiya, H. Ohta, T. Uruga, M. Hirano and H. Hosono, Phys. Rev. B: Condens. Matter Mater. Phys., 2007, 75, 035212.

11 L. Lan, N. Xiong, P. Xiao, M. Li, H. Xu, R. Yao, S. Wen and J. Peng, Appl. Phys. Lett., 2013, 102, 242102.

12 H. Nakanotani, M. Yahiro, C. Adachi and K. Yano, Appl. Phys. Lett., 2007, 90, 262104.
13 J. Smith, A. Bashir, G. Adamopoulos, J. E. Anthony, D. D. C. Bradley, R. Hamiltion, M. Heeney, I. McCulloch and T. D. Anthopoulos, Adv. Mater., 2010, 22, 3598.

14 C. Yang, Y. Kwack, S. H. Kim, T. K. An, K. Hong, S. Nam, M. Park, W. Choi and C. E. Park, Org. Electron., 2011, 12, 411.

15 B. Kim, S. Jang, M. L. Geier, P. L. Prabhumirashi, M. C. Hersam and A. Dodabalapur, Appl. Phys. Lett., 2014, 104, 62101.

16 J. Shi, H. Wang, D. Song, H. Tian, Y. Geng and D. Yan, Adv. Funct. Mater., 2007, 17, 397.

17 E. J. Meijer, D. M. Deleeuw, S. Setayesh, E. V. Veenendaal, B. H. Huisman, P. W. Blom, J. C. Hummelen, U. Scherf and T. M. Klapwijk, Nat. Mater., 2003, 2, 678.

18 K. Baeg, D. Khim, S. Jung, M. Kang, I. You, D. Kim, A. Facchetti and Y. Noh, Adv. Mater., 2012, 24, 5433.

19 I. Meager, M. Nikolka, B. C. Schroeder, C. B. Nielsen, M. Planells, H. Bronstein, J. W. Rumer, D. I. James, R. S. Ashraf, A. Sadhanala, P. Hayoz, J. Flores, H. Sirringhaus and I. McCulloch, Adv. Funct. Mater., 2014, 24, 7109.

20 J. Y. Choi, W. Kang, B. Kang, W. Cha, S. K. Son, Y. Yoon, H. Kim, Y. Kang, M. J. Ko, H. J. Son, K. Cho, H. J. Cho and B. Kim, ACS Appl. Mater. Interfaces, 2015, 7, 6002.

21 Y. Li, L. Lan, P. Xiao, Z. Lin, S. Sun, W. Song, E. Song, P. Gao, P. Zhang and J. Peng, J. Mater. Chem. C, 2016, 4, 2072.

22 S. Sun, L. Lan, P. Xiao, Z. Lin, H. Xu, M. Xu and J. Peng, RSC $A d v .$, 2015, 5, 15695.

23 J. Veres, S. Ogier and G. Lloyd, Chem. Mater., 2004, 16, 4543. 24 X. Cheng, M. Caironi, Y. Y. Noh, J. Wang, C. Newman, H. Yan, A. Facchetti and H. Sirringhaus, Chem. Mater., 2010, 22, 1559.

25 J. Veres, S. D. Ogier, S. W. Leeming, D. C. Cupertino and S. MohialdinKhaffaf, Adv. Funct. Mater., 2003, 13, 199.

26 D. K. Owens and R. C. Wendt, J. Appl. Polym. Sci., 1969, 13, 1741.

27 F. M. Fowkes, J. Phys. Chem., 1963, 67, 2538.

28 B. Singh, P. Senkarabacak, N. S. Sariciftci, A. Tanda, C. Lackner, R. Hagelauer and G. Horowitz, Appl. Phys. Lett., 2006, 89, 033512.

29 M. J. An, H. S. Seo, Y. Zhang, J. D. Oh and J. H. Choi, Appl. Phys. Lett., 2010, 97, 023506.

30 J. D. Oh, J. W. Kim, D. K. Kim and J. H. Choi, Org. Electron., 2016, 30, 131. 